\title{
Cuotas de emisión de gases de efecto invernadero y ayudas públicas en el Derecho Comunitario Europeo(*)
}

\author{
Bernard-Frank Macera \\ Profesor Titular de Derecho Administrativo \\ (Universidad de Valladolid)
}

\section{INTRODUCCIÓN}

Con el fin de alcanzar determinados objetivos de interés general, los Poderes públicos acuden con frecuencia a diversos instrumentos a los que se vincula la presunción técnico-jurídica de que se trata de ayudas públicas (con todas las implicaciones que eso conlleva en el terreno de su tratamiento legal ${ }^{1}$ ), porque tienen como objeto directo la concesión de beneficios económicos. Es el caso, por ejemplo, de las subvenciones (como "punctum saliens" de la actividad administrativa dispensadora de ayudas ${ }^{2}$ ); de la exoneración, deducción y devolución total o parcial de impuestos y tributos; de la cesión de inmuebles a título gratuito o en condiciones favorables; de los créditos y préstamos en condiciones de ventaja; de la desgravación y percepción diferida de cotizacio-

(*) El presente trabajo se redactó inicialmente como contribución al libro: S. Aníbarro Pérez; I. Caro-Patón Carmona; B.F. Macera; E. Martínez Pérez; M. Pardo Alvarez; I. Sanz Rubiales (Director), El mercado de derechos a contaminar (régimen jurídico-público del mercado comunitario de derechos de emisión en España), Ed. Lex Nova, Valladolid, 2007.

${ }^{1}$ Una de las implicaciones más importantes de la consideración de una medida nacional como ayuda pública es, desde luego, como veremos a lo largo del presente trabajo, su necesario sometimiento a las reglas previstas por el Tratado constitutivo de la Comunidad Europea, en sus artículos 87 a 89 .

${ }^{2}$ Debemos a MARTÍNEZ LÓPEZ-MUÑZ la construcción teórica del modo de actividad administrativa del que las ayudas públicas son el objeto o instrumento principal. En especial, vid. su trabajo: «La actividad administrativa dispensadora de ayudas y recompensas: una alternativa conceptual al fomento en la teoría de los modos de acción de la Administración pública», en vol. col., Libro Homenaje al Profesor José Luis Villar Palasí, Ed. Civitas, Madrid, 1989. 
nes o cualquier tipo de aligeramiento de cargas sociales; y un largo etcétera ${ }^{3}$. Sin embargo, cabe advertir que esta presunción no es "absoluta" sino relativa", por lo que existe siempre la posibilidad de destruirla ${ }^{4}$. En efecto, la consideración de dichas técnicas como ayuda pública desaparecerá en el supuesto en que no estén reunidos ciertos elementos definitorios, entre los que destaca, como veremos a lo largo del presente trabajo, el de la selectividad. Dicho de manera esquemática, se suele considerar que una medida que favorece a colectivos indeterminados no constituye, en sentido estricto, una ayuda pública, aunque proporcione de manera directa y evidente una ventaja económica a sus destinatarios. Tal es el caso, por ejemplo, de los beneficios fiscales a favor de productos nacionales en general ${ }^{5}$.

Otros instrumentos, a priori, no pueden equipararse presuntamente a ayudas públicas, porque, a diferencia de los anteriores, no tienen como objeto directo la dispensación de auténticos beneficios económicos. Es el caso, por ejemplo, en principio, de las tomas de participaciones accionariales en empresas por parte de los Poderes públicos. Sin embargo, de la misma manera que se puede destruir la presunción de ayuda pública de todas aquellas medidas por las que se otorgan directamente dichos beneficios, es posible destruir también la presunción contraria que se aplica a todos aquellos instrumentos que no tienen tal objeto. Volviendo al ejemplo antes mencionado, se considera que si la toma de participación se realiza en unas condiciones

\footnotetext{
${ }^{3}$ Como se puede observar y como resulta conocido, las ayudas públicas no están vinculadas a ninguna figura jurídica determinada sino que son susceptibles de revestir innumerables formas, cuyo elenco, como advierte COLLIARD («Les aides accordées par les États», en vol. col., Droit des communautés européennes, Ed. Larcier, Bruxelles, 1969, pág. 864), no puede pretender ser exhaustivo. Sobre este aspecto, vid. por ejemplo, B.F. MACERA, El deber industrial de respetar el ambiente (análisis de una situación pasiva de Derecho público), Ed. Marcial Pons, Madrid-Barcelona, 1998, págs. 334-335.

${ }^{4}$ Pensando en el lector ajeno al mundo del Derecho, recordaremos que las «presunciones relativas», a diferencia de las «absolutas», sólo se mantienen mientras no se produzca prueba en contrario.

${ }^{5}$ Ténganse en cuenta al respecto, en la jurisprudencia comunitaria, las Sentencias del Tribunal de Justicia de la Comunidad Europa de 22 de marzo de 1977, «Ianelli/Meroni», y de 27 de mayo de 1981, «Essevi et Salengo». En la doctrina, vid. por ejemplo, J.L. MARTÍNEZ LÓPEZ-MUÑIZ, «Ayudas públicas», en vol. col., Derecho europeo de la competencia (antitrust e intervenciones públicas), coord.: L.A. Velasco San Pedro, Ed. Lex Nova, Valladolid, 2005, págs. 502-503. Vid. también, D.H. SCHEUING, Les aides financières publiques, Ed. Berger-Levrault, París, 1974; G. FERNÁNDEZ FARRERES, El régimen de las ayudas estatales en la Comunidad Europea, Ed. Civitas, Madrid, 1993, págs. 62 y ss.
} 
económicas ventajosas (para la empresa correspondiente) que ningún inversor privado estaría dispuesto a ofrecer, estamos en presencia una ayuda pública. La inversión realizada por los Poderes públicos, en efecto, libera a su destinatario de costes que las demás empresas tienen que asumir por sí mismas en un sistema basado en la libre competencia. Y la toma de participación no responde a una lógica empresarial de mercado sino que se realiza por otros motivos ${ }^{6}$.

En este trabajo, debemos preguntarnos a cuál de las dos categorías pertenece la asignación a empresas de cuotas de emisión a la atmósfera, como medida que se contempla, en nuestro país, en la Ley 1/2005, de 9 de marzo, por la que se regula el régimen de comercio de derechos de emisión de gases de efecto invernadero (por la que se transpone al ordenamiento español la Directiva 2003/87/CE del Parlamento Europeo y del Consejo, de 13 de octubre de 2003, por la que se establece un régimen para el comercio de derechos de emisión de gases de efecto invernadero en la Comunidad -en adelante, "la Directiva de 2003"-) y que se instrumenta a través del Plan Nacional de Asignación aprobado por el Real Decreto 1866/2004, de 6 de septiembre (Boletín Oficial del Estado del 7). En nuestra opinión, estamos ante una especie del segundo género. Para intentar demostrarlo, vamos a seguir un razonamiento eminentemente sencillo si tenemos en cuenta las reflexiones que acabamos de formular en la presente introducción. En concreto, en un primer momento, nos proponemos explicar por qué razones consideramos aplicable a la asignación de cuotas de emisión a la atmósfera la presunción de que no se trata de una ayuda pública (II), antes de subrayar, en un segundo momento, el carácter relativo de dicha presunción y de explicar, como consecuencia de ello, que es susceptible de destruirse en determinadas circunstancias (III).

\footnotetext{
${ }^{6} \mathrm{El}$ criterio llamado del «inversor privado» o del «operador privado», que sirve para proporcionar indicios decisivos de la presencia de ayudas públicas en ciertas actuaciones económico-financieras de los Poderes públicos, ha sido elaborado por la jurisprudencia comunitaria. Vid. entre otras las Sentencias del Tribunal de Justicia de la Comunidad Europea de 29 de abril de 1999, «España/Comisión», de 29 de junio de 1999, «DM Transport», de 8 de julio de 2004, «Glaswerke Ilmenau/Comisión», etc.
} 


\section{UNA PRESUNCIÓN: LA ASIGNACIÓN DE CUOTAS NO CONS- TITUYE A PRIORI UNA AYUDA PÚBLICA EN EL SENTIDO DEL DERECHO COMUNITARIO}

Nuestra postura se fundamenta en un análisis que consiste, en un primer momento, en resaltar los caracteres generales de las ayudas públicas (1) para contrastarlos, en una segunda etapa, con los que definen el sistema de asignación a las empresas de cuotas de emisión (2).

1) Rasgos generales de las ayudas públicas

La definición rigurosa del concepto de ayuda pública no es tarea fácil. Estamos ante una noción "camaleón”, que se utiliza con un alcance semántico polivalente e incluso contradictorio. Ahora bien, el Derecho comunitario, precisamente porque ha prestado una particular atención a ciertas formas generales de intervención pública que pueden tener una incidencia en el funcionamiento del mercado europeo -entre ellas, las ayudas públicas-, nos proporciona una herramienta que reviste una gran utilidad en cualquier intento de definición y de la que cabe extraer principios y reglas generalizables a nuestro escenario jurídico, ante la ausencia de concepción precisa en el Derecho interno. Hablamos aquí, con toda evidencia, del artículo 87.1 del Tratado constitutivo de la Comunidad Europea (en adelante, TCE), que hace referencia, precisamente, a "las ayudas otorgadas por los Estados o mediante fondos estatales, bajo cualquier forma, que falseen o amenacen falsear la competencia, favoreciendo a determinadas empresas o producciones". Ahora bien, como ha resaltado una parte de la doctrina, dicho precepto no es suficiente para lograr una definición satisfactoria, aunque sus carencias pueden explicarse por la perspectiva reguladora que le es propia ${ }^{7}$. Por ello, como vamos a poder ilustrar a continuación, es aconsejable acudir a diversos pronunciamientos jurisprudenciales (en particular, del Tribunal de Justicia de la Comunidad Europea) y doctrinales que precisan e interpretan la noción contenida en el $\mathrm{TCE}^{8}$, así como a algunos textos de la Comisión (en particular, el Vademecum Community rules on State Aid ${ }^{9}$ ).

\footnotetext{
${ }^{7}$ Por ejemplo, vid. M. VACCA, «Gli aiuti di Stato alle imprese. Considerazioni introduttive», Rivista di Diritto Europeo, núm. 1/1993, pág. 5.

${ }^{8}$ Hemos de referirnos, de nuevo, a MARTÍNEZ LÓPEZ-MUÑIZ. En su trabajo ya citado «La actividad administrativa...» (págs. 758 y ss.), dicho autor define las ayudas como actos del Poder público (en principio, de la Administración) consistentes en «el otorgamiento directo o indirecto de bienes o derechos a determinados administrados -privados o públicos-, con carácter no devolutivo y por razón de ciertas actividades que les son propias, aún por desarrollar, quedando afectados a su realización».

${ }^{9}$ European Commission, Bruselas, 2003, pág. 3.
} 
En primer lugar, para que una medida pueda considerarse como una ayuda pública, ello es evidente y se desprende de la expresión utilizada por el precepto antes citado ("las ayudas otorgadas por los Estados o mediante fondos estatales"), debe ser imputable a un Poder público, en el sentido en que ha de nutrirse de recursos financieros (cualquiera que sea su naturaleza, como bien, derecho o ventaja) sobre los que dicho Poder público esté en condición de ejercer una potestad de afectación, cualquiera que sea el cauce formal utilizado ("bajo cualquier forma" $\left.{ }^{\text {"0 }}\right)^{11}$. Como indica el Tribunal de Justicia de la Comunidad Europea en su Sentencia de 16 de mayo del 2000, "Francia/Ladbroke Racing y Comisión", "el artículo 87, apartado 1, comprende todos los medios económicos que el Estado puede efectivamente utilizar para apoyar a las empresas. El hecho de que estos medios permanezcan constantemente bajo control público y, por tanto, a disposición de las autoridades nacionales competentes, basta para calificarlos de fondos estatales y para que dicha medida esté comprendida dentro del ámbito de aplicación del artículo 87, apartado 1"12. Hemos de advertir que el Tribunal de Justicia de la Comunidad Europea, a pesar del carácter abierto de dicha definición, parece mantener una línea restrictiva en lo que se refiere al origen real de la aportación recibida por el destinatario de la ayuda, lo cual ha provocado numerosas críticas. Más precisamente, según el Tribunal de Luxemburgo, las ayudas públicas, para llegar a serlo efectivamente, deben constituir una carga para los Poderes públicos, por proceder de alguna manera de sus recursos o de los que de otro modo tendrían que entrar bajo su titularidad y, por tanto, bajo su disponibilidad.

En segundo lugar, como estipula el antes citado artículo 87.1 del TCE y como ya hemos adelantado en la introducción del presente trabajo, las ayudas públicas han de dirigirse a favorecer a determinados sujetos o actividades de estos sujetos, es decir, con otras palabras, deben revestir un cierto grado de selectividad. Se distinguen así de las medidas generales de fomento, estímulo o favorecimiento dirigidas a colectivos indeterminados o a todo un determinado sector ${ }^{13}$. Como consecuencia de ello, si una medida favorable beneficia a

\footnotetext{
${ }^{10}$ Nos remitimos aquí a la nota núm. 3.

11 Vid. sobre este criterio, J.L. MARTÍNEZ LÓPEZ-MUÑIZ, «Ayudas públicas», cit., págs. 493 y ss.

12 Téngase en cuenta también, más recientemente, la Sentencia del Tribunal de Justicia de la Comunidad Europea de 29 de abril de 2003, «Grecia/Comisión».

13 Téngase presente la Sentencia del Tribunal de Justicia de la Comunidad Europea de 15 de julio de 2004, «Pearle y otros».
} 
todas las empresas y no sólo a ciertos centros de producción, no se trata de una ayuda pública propiamente dicha sino de lo que la doctrina denomina una "medida económica general" ${ }^{4}$. Ahora bien, como advierte el Tribunal de Justicia de la Comunidad Europea en su Sentencia de 26 de septiembre de 1996, "Francia/Comisión", "la jurisprudencia ya ha precisado que incluso intervenciones que, a primera vista, son aplicables a la generalidad de las empresas pueden caracterizarse por una cierta selectividad y, por consiguiente, ser consideradas como medidas destinadas a favorecer a determinadas empresas o producciones". Así sucede, en particular, como ilustra el pronunciamiento citado, "cuando la Administración que debe aplicar la norma general disponga de una facultad discrecional en lo que respecta a la aplicación del acto"15.

Finalmente, en tercer lugar, nos parece importante resaltar, volviendo al contenido literal del precepto aquí comentado, que las ayudas públicas, para ser consideradas como tales, deben "favorecer" a sus destinatarios (aunque dicho aspecto suele contemplarse no como un elemento definitorio independiente sino vinculado al criterio antes mencionado de la selectividad). Se deduce de ello que sólo pueden ser consideradas como ayudas públicas aquellas medidas cuyo efecto directamente pretendido sea favorecedor y que produzcan un beneficio económico y un "enriquecimiento" relativo o una mejora de la situación económica del destinatario ${ }^{16}$. En nuestra opinión, no pueden constituir por sí mismas actos de limitación o constrictivos, entre los que se incluyen, por ejemplo, las reglamentaciones técnicas o, también, los precios fijados por vía administrativa para proteger a los usuarios o consumidores, etc. ${ }^{17}$

Para no dejar cabos sueltos, tenemos que detenernos brevemente en la última porción de frase del artículo 87.1 del TCE que nos queda por mencionar y que viene constituida por la referencia a las ayudas "que falseen o amenacen falsear la competencia". Ahora bien, hemos de advertir que no se trata de una precisión con vocación definitoria. Una medida favorable no es una ayuda públi-

\footnotetext{
${ }^{14}$ Sobre este tipo de medidas, vid. por ejemplo, M.G BRIOTTI, «Politiche industriali e trasferimenti pubblici alle imprese nel mercato unico europeo», Rivista di Diritto Europeo, núm. 1/1995, págs. 126 y ss.

15 Vid. también las Sentencias del Tribunal de Justicia de la Comunidad Europea de 1 de diciembre de 1998, «Ecotrade», y de 17 de junio de 1999, «Piaggio».

16 Vid. B.F. MACERA, El deber industrial..., cit., pág. 333.

17 Vid. en tal sentido, J.L. MARTÍNEZ LÓPEZ-MUÑIZ, «Ayudas públicas», cit., págs. 502-503.
} 
ca porque falsea o amenaza falsear la competencia. Lo es únicamente porque reúne las características y criterios antes mencionados del origen público, de la selectividad y del carácter favorecedor para su destinatario. La incidencia (real o potencial) en los mecanismos que articulan el libre mercado no es más que un efecto que pueden tener y que, en principio, tienen las ayudas públicas y que, por ello, si la perturbación que conllevan traspasa los límites territoriales nacionales, hace que tengan que someterse a una regulación estricta esencialmente basada, como veremos un poco más adelante, en el principio de incompatibilidad con el Derecho comunitario de la competencia.

\section{2) Su aplicación al sistema de asignación de cuotas}

La aplicación de los tres criterios antes analizados al sistema de asignación por los Poderes públicos a las empresas de cuotas de emisión a la atmósfera (que se instrumenta a través de los Planes Nacionales de Asignación), como ya hemos adelantado en la introducción, no deja lugar a incertidumbres. No estamos ante ayudas públicas. Solamente una de dichas condiciones plantea dudas acerca de la cuestión de saber si viene cumplida por el sistema aquí analizado. Nos referimos al criterio del origen público de la medida contemplada. En realidad, el interrogante reside en el hecho de que, si bien la asignación de cuotas constituye una medida inequívocamente pública (que conlleva ejercicio de autoridad) ${ }^{18}$ e imputable a los Estados ${ }^{19}$, no parece que podamos hablar de una auténtica transferencia de recursos públicos (como condición que viene exigida, como antes hemos visto, por la jurisprudencia del Tribunal de Justicia de la Comunidad Europea). Según algunos autores, sí es el caso, porque los títulos de emisión tienen un valor de mercado y la Administración renuncia a unos ingresos que obtendría en el caso de que los derechos fueran cedidos de manera onerosa ${ }^{20}$. Hemos de precisar, en efecto, que el sistema se

18 Vid. en tal sentido, I. SANZ RUBIALES, «Una aproximación al nuevo mercado de derechos de emisión de gases de efecto invernadero", Revista Española de Derecho Administrativo, núm. 125, enero-marzo 2005, pág. 54.

19 Téngase presente el Informe sobre la política de concurrencia para 2004, SEC (2005) 805 final, núms. 518 y 519.

${ }^{20}$ Vid. por ejemplo, F. ROSEMBUJ GONZÁLEZ-CAPITEL, El precio del aire. Aspectos juridicos del mercado de derechos de emisión, Ed. El fisco, Barcelona, 2005. Vid. también, S. ROUSSEAUX, «L'allocation des quotas d'émission de gaz à effet de serre: un aspect déterminant du futur marché européen», Revue du Marché Commun et de l'Union Européenne, núm. 484, enero 2005, pág. 33. Según esta autora (ibid.), «les États renoncent à des ressources qui pourraient être versées à leur budget dans le cadre d'une allocation à titre onéreux. L'allocation des quotas à titre gratuit constitue dès lors une aide accordée par des ressources d'État» (loc. cit.). 
articula inicialmente y de manera casi exclusiva con arreglo a un método de asignación gratuita de las cuotas. La Comisión Europea también se pronuncia en tal sentido. Para dicho órgano, un derecho de emisión constituye en cierta medida un activo intangible cuyo valor viene determinado por el mercado, por lo que el hecho de que el Estado proceda a su asignación a las empresas emisoras de forma gratuita equivale al otorgamiento de una ventaja que deriva de una transferencia de recursos estatales ${ }^{21}$. Ahora bien, conviene no perder de vista, a nuestro parecer, que si bien es verdad que los títulos de emisión poseen un cierto valor mercantil, su asignación gratuita no puede considerarse como una auténtica opción para los Poderes públicos nacionales, sino que se trata de una exigencia impuesta por el Derecho comunitario europeo ${ }^{22}$. El Estado, por tanto, en rigor, no renuncia a unos ingresos cuando distribuye gratuitamente las cuotas a las empresas interesadas, sino que no hace más que cumplir los mandatos establecidos a nivel comunitario ${ }^{23}$.

Una vez llegados a este punto, tenemos que advertir que, con independencia de la postura que sea posible adoptar en relación con dicha cuestión, aportar una respuesta clara e inequívoca no es absolutamente decisivo. Como hemos advertido unas líneas atrás, los otros dos elementos que condicionan la existencia de ayudas públicas, en nuestra opinión, no están reunidos. Y ello es suficiente para que podamos mantener la conclusión que, desde el principio del trabajo, venimos formulando.

${ }^{21}$ La Comisión formuló dicho planteamiento, por ejemplo, al examinar las condiciones del mercado danés de derechos de emisión de $\mathrm{CO}_{2}$, en su Decisión de 29 de marzo de 2000 (la primera relativa a este tema), si bien no se opuso a la creación de este mercado (N 653/1999, Diario Oficial de la Comunidad Europea serie C de 11 de noviembre de 2000). Recordemos que los Poderes públicos daneses, muy tempranamente, crearon un mercado de derechos de emisión que incluía de manera exclusiva a las instalaciones generadoras de energía eléctrica y cuya duración cubría los años 2001-2003. A las instalaciones afectadas se les donaban los títulos correspondientes de modo gratuito sobre la base de sus emisiones históricas durante el periodo 1994-1998.

${ }^{22} \mathrm{El}$ artículo 10 de la Directiva de 2003 establece literalmente, sin dejar ninguna opción a los Estados miembros en cuanto se refiere al método empleado, que "Para el periodo de tres años que comenzará el 1 de enero de 2005 los Estados miembros asignarán gratuitamente al menos el $95 \%$ de los derechos de emisión". El mismo precepto añade que "Para el periodo de cinco años que comenzará el 1 de enero de 2008, los Estados miembros asignarán gratuitamente al menos el 90 \% de los derechos de emisión".

${ }^{23}$ Vid. en tal sentido, por ejemplo, M. RodI, «Legal aspects of the European Emissions Trading Scheme», en vol. col., Emissions Trading for Climate Policy. US and European Perspectives (ed.: B. Hansjürgen), Cambridge University Press, 2005, pág. 187. 
En cuanto al criterio de la selectividad, por un lado, tenemos que partir de la premisa de que los Planes Nacionales de Asignación, porque así lo exige la Directiva (en su artículo 9 y, más precisamente, en su Anexo III $^{24}$ ), no deben distinguir entre empresas o sectores de modo que se favorezca indebidamente a determinadas empresas o actividades, tanto desde el punto de vista de la aplicación de las cuotas como desde la perspectiva de su cuantía ${ }^{25}$. Dicho con otras palabras, los Planes, en principio, no pueden establecer discriminaciones entre los destinatarios de la asignación que se encuentran en condiciones más o menos similares o que realizan las mismas o análogas actividades, sino que deben basarse en criterios estrictamente objetivos que permitan un trato justo y equitativo de las empresas ${ }^{26}$. El reparto de cuotas, por tanto, si se realiza de acuerdo con dichas reglas básicas, sencillamente, no reviste un carácter selectivo.

Por lo que se refiere al criterio del carácter favorecedor o beneficioso para el destinatario de la medida, por otro lado, no debemos olvidar que la asignación de cuotas no hace sino reflejar lo que venía o vendría definido por normas o por actos administrativos de policía en el marco de un sistema clásico de protección ambiental basado en la coacción ${ }^{27}$, con unos techos de emisión previamente fijados por los Poderes públicos. Y ello es coherente con los objetivos que, en el fondo, se procura alcanzar con el establecimiento del sistema de cuotas. En efecto, como reconoce la propia Comisión Europea, "el comercio de derechos de emisión no implica nuevos objetivos ambientales sino que facilita el cumplimiento menos costoso de los objetivos vigentes" 28 . Además, como destaca SANZ Rubiales, la regla de la gratuidad, que -inicialmente-

\footnotetext{
${ }^{24}$ Dicho Anexo establece los «criterios aplicables a los Planes Nacionales de Asignación contemplados en los artículos 9, 22 y 30» de la Directiva de 2003.

25 Punto 5 del Anexo III de la Directiva de 2003.

26 Vid. en contra, S. RoussEaux, «L'allocation...», cit., pág. 33.

27 Vid. M. RoDI, «Legal aspects...», cit., pág. 181.

28 Preguntas y respuestas sobre el comercio de derechos de emisión y los planes nacionales de asignación, Comisión Europea, MEMO/04/44, Bruselas, 4 de marzo de 2004, punto 1. La Comisión (ibid., loc. cit.) afirma a continuación que "el hecho de que las empresas participantes puedan comprar o vender derechos de emisión significa que podrán alcanzarse los objetivos al menor coste. Si no se hubiera adoptado el régimen de comercio de derechos de emisión, hubieran tenido que aplicarse otras medidas más costosas".
} 
rige en materia de $\operatorname{cuotas}^{29}$, no altera en modo alguno esta circunstancia sino que no hace más que mantener la situación vigente hasta el momento ${ }^{30}$. Dicho con otras palabras, en suma, las empresas reciben cuotas para emitir $\mathrm{CO}_{2}$ en las mismas condiciones que lo que venían haciendo, esto es, en principio, con unos límites predeterminados y sin pagar por ello. No existe, en sentido estricto, un auténtico beneficio para las empresas receptoras de las cuotas de emisión. Las únicas diferencias que introduce el sistema de asignación con respecto a la situación anterior se sitúan en las consecuencias vinculadas a los resultados empresariales en materia de emisiones: las empresas que superan los límites de contaminación predeterminados deben pagar para adquirir en el mercado las cuotas que les faltan, bajo amenaza de sanción, y, a la inversa, las que reduzcan sus emisiones con respecto a los citados límites podrán obtener una rentabilidad económica resultante de la venta de las cuotas no utilizadas. Es evidente que la situación de las primeras no refleja la existencia de una ventaja financiera, todo lo contrario. Y por lo que se refiere a las segundas, conviene aclarar que el beneficio económico que deriva del producto de la venta de cuotas no es la consecuencia directa de la asignación realizada por los Poderes públicos sino que es el resultado de un esfuerzo hecho por la empresa para reducir las emisiones de $\mathrm{CO}_{2}$, un esfuerzo que se concreta probablemente en inversiones costosas. Otra cosa es que el excedente de cuotas no pueda considerarse como el fruto de esta "capacidad" de la empresa sino como el resultado de una asignación generosa por parte de los Poderes públicos. En tal caso, como veremos un poco más adelante, sí estaríamos ante una ayuda pública. Pero, en principio, si las autoridades nacionales respetan los mandatos establecidos en la Directiva de 2003, no deberíamos encontrarnos en tal circunstancia.

Hemos de advertir que la tesis que aquí defendemos difiere sustancialmente de la que sostienen la Comisión Europea y numerosos autores. El razonamiento que sirve de base a la postura mencionada puede resumirse a través de las palabras que RoussEAux dedica a esta problemática. En síntesis, se afirma que en la medida en que los titulares de las instalaciones sometidas a la

29 Téngase presente, de nuevo, el ya citado artículo 10 de la Directiva de 2003.

${ }^{30}$ I. SANZ RUBIALES, «Una aproximación...», cit., pág. 41. Como es evidente, en un sistema que privilegiara la adquisición onerosa de las cuotas (de acuerdo con el mecanismo de la subasta), la ausencia de carácter favorecedor sería aún más evidente. En efecto, dicho sistema obligaría a las empresas a pagar anticipadamente por algo (realizar emisiones dentro de los límites establecidos por el ordenamiento jurídico) que antes era gratuito. 
Directiva de 2003 reciben gratuitamente cuotas que tienen un valor económico -porque pueden ser compradas y vendidas-, la asignación de cuotas es una ayuda de Estado, porque supone una ventaja económica" ${ }^{1}$, una ventaja que resulta, además, de una disminución o eliminación de las cargas que habrían de soportar si tuvieran que comprar las cuotas con arreglo a un sistema de subasta $^{32}$. No estamos de acuerdo con dicho pronunciamiento, básicamente por dos razones. En primer lugar, no nos parece que el argumento del valor económico del bien o del derecho transmitido (subrayado también por la Comisión $^{33}$ ) sea decisivo a la hora de calificar la medida correspondiente de otorgamiento como una ayuda pública. Basta pensar en el ejemplo de las concesiones administrativas. Estos títulos, bajo ciertas condiciones, pueden ser objeto de transmisión onerosa por parte de sus titulares, por lo que se puede considerar que tienen un valor económico. Pero ¿cabe afirmar por ello que el otorgamiento de una concesión administrativa constituye necesariamente una ayuda pública? No creemos que se pueda llegar a esta conclusión. En segundo lugar, tampoco nos parece acertado sostener que la asignación gratuita de cuotas es una ayuda pública sencillamente porque comporta una ventaja económica que deriva del hecho de que se libera a las empresas de la carga de tener que comprarlas. Esta afirmación sería exacta si el mecanismo de la subasta (como forma o cauce de adquisición onerosa) fuese la regla general de asignación de las cuotas. Pero, como ya hemos podido comprobar, no es el caso ${ }^{34}$. Defender lo contrario constituye un auténtico sofisma tan poco convincente como, por ejemplo, el que consiste en afirmar que el hecho de no someter una industria a ciertas normas nuevas (que no han entrado a formar parte del ordenamiento jurídico vigente) constituye una exención o dispensa que equivale a una ayuda pública. En este caso, de modo similar a lo que ocurre con la problemática a la que van dedicas las presentes líneas, únicamente podríamos hablar de la existencia de una ayuda pública si las normas en cuestión estuvieran en vigor y se aplicaran a todas las empresas del sector.

${ }^{31}$ Téngase presente en tal sentido la antes citada Decisión de la Comisión de 29 de marzo de 2000 N 653/1999 sobre el mercado danés de derechos de emisión de $\mathrm{CO}_{2}$ (Diario Oficial de la Comunidad Europea serie C de 11 de noviembre de 2000).

${ }^{32}$ S. ROUSSEAUX, «L'allocation...», cit., págs. 32-33.

33 Vid. supra la postura de dicho órgano comunitario acerca de la cuestión que consiste en saber si la asignación gratuita de cuotas constituye una transferencia de recursos públicos.

${ }^{34}$ Cabe referirse, de nuevo, al artículo 10 de la Directiva de 2003, que se transcribe íntegramente en la nota núm 22. 
En resumen, cabe reiterar, con SANZ RUBIALES, que si los Estados se acomodan a los criterios de asignación marcados por la Directiva de 2003, sus Planes de Asignación no constituyen, per se, ayudas de Estado según el artículo 87.1 del TCE ${ }^{35}$. Ahora bien, como vamos a poder comprobar a renglón seguido y como ya hemos advertido en la introducción, es posible destruir dicha presunción.

\section{SU RELATIVIDAD: LA ASIGNACIÓN DE CUOTAS PUEDE CONS- TITUIR UNA AYUDA PÚBLICA EN DETERMINADOS CASOS}

1) Una premisa: la posible presencia en los Planes de elementos característicos de las ayudas públicas

Hemos advertido antes que el hecho de que un instrumento o una técnica no pueda calificarse inicialmente como una ayuda pública no significa que no pueda convertirse, en determinadas circunstancias, en una medida de este tipo. Es precisamente lo que ocurre con la asignación de cuotas de emisión contenida en los Planes Nacionales. En efecto, como ha destacado la Comisión Europea, es posible que dichos Planes reúnan ciertos criterios característicos de las ayudas públicas, en el sentido del artículo 87.1 del TCE.

Desde un punto de vista general, ello ocurrirá en el supuesto en que el Plan distinga entre empresas o sectores de modo que favorezca indebidamente a determinadas empresas o actividades, lo que se manifestará esencialmente en el establecimiento de criterios de asignación no objetivos o no transparentes.

35 I. SANZ RUBIALES, «Una aproximación...», cit., págs. 56-57. Vid. también, M. RODI, «Legal aspects...», cit., pág. 187. El hecho de que las normas contables españolas (concretamente, la Resolución de 8 de febrero de 2006, del Instituto de Contabilidad y Auditoría de Cuentas, por la que se aprueban normas para el registro, valoración e información de los derechos de emisión de gases de efecto invernadero) contemplen las transferencias de derechos de emisión a las empresas como subvenciones públicas (lo que explica su reflejo en balance como inmovilizados inmateriales) no debe considerarse como un dato decisivo en el análisis que estamos llevando a cabo. Al contrario, dicho planteamiento ha de interpretarse como una solución pragmática motivada exclusivamente por la ausencia de alternativas más ajustadas a la auténtica naturaleza de su objeto. En otros países, como, por ejemplo, en Bélgica, las normas contables dejan a las empresas un cierta margen de libertad a la hora de dar reflejo en sus cuentas anuales a la transmisión de derechos de emisión. Vid. en relación con este tema el Dictamen de la Commission des normes comptables de Belgique 179-1 (agosto 2005) relativo al tratamiento contable de los cuotas de emisión de gases con efecto invernadero. 
Desde un punto de vista más específico, cabe destacar, sin pretensión de exhaustividad, que la existencia de ayudas públicas podrá ser la consecuencia, por ejemplo, del establecimiento en los Planes de métodos de cálculo de emisiones que permitan a las autoridades nacionales asignar más cuotas de las estrictamente necesarias, lo que otorgaría a las empresas beneficiarias la posibilidad de extraer un beneficio económico de la venta de los títulos sobrantes ${ }^{36}$. Otra posibilidad es que los Planes no respeten las condiciones de equidad en relación con las empresas (nacionales o extranjeras) que deseen entrar y establecerse en el mercado, en particular en lo que se refiere al método o sistema de asignación de las cuotas.

2) Una consecuencia lógica: el sometimiento de los Planes a las reglas de Derecho comunitario relativas a las ayudas públicas

En el supuesto en que reúnan las características esenciales de las ayudas públicas, los Planes han de someterse a las reglas que dedica el TCE a tales medidas de intervención. Dicha regulación, como es conocido, se articula esencialmente en torno a un presupuesto básico (a), un principio general (b) y a unas excepciones (c).

a) Un presupuesto básico: la obligación estatal de notificación

En principio, como establece el artículo 88 del TCE, las medidas que contienen presuntamente ayudas públicas deben ser notificadas por los Estados miembros a la Comisión Europea, precisamente para permitir a este órgano ejercer las facultades de control que le atribuye el mismo precepto ${ }^{37}$. Ahora

\footnotetext{
36 Según la Comisión Europea (Preguntas y respuestas..., cit.), una asignación excesiva puede deberse a varias causas. «En primer lugar, a que un Estado miembro no explique cómo se cumplirá el objetivo de Kioto durante el período 2008-2012, sino que deja una laguna que debe llenarse con medidas que van a determinarse posteriormente. En segundo lugar, a que un Estado miembro declare su intención de adquirir créditos de Kioto, pero no presenta medidas creíbles ni fiables para llevar a cabo esas adquisiciones. En tercer lugar, a que un Estado miembro base su plan en previsiones (incluidas las tasas de crecimiento económico y de aumento de emisiones) incoherentes y excesivas respecto a las estimaciones de crecimiento oficiales del mismo Estado miembro o de otras fuentes imparciales» (punto 7).

37 Dicho órgano queda investido de potestades de investigación y de control sobre todos los proyectos de concesión de ayudas (control preventivo) y sobre los sistemas o planes de dispensación ya existentes o realizados (control permanente). Además, puede exigir la supresión o modificación de las ayudas proyectadas u otorgadas. Conviene añadir, finalmente, que entre los medios al alcance de la Comisión Europea para garantizar el respeto de las obligaciones impuestas
} 
bien, en relación con los Planes nacionales de asignación, tenemos que preguntarnos si esta obligación viene o no cumplida, en realidad, con la notificación o comunicación previa a la Comisión que se contempla, como trámite del procedimiento de aprobación y elaboración de dichos Planes, por los apartados segundo y tercero del artículo 9, así como por el artículo 23 de la Directiva de $2003^{38}$.

En nuestra opinión, si bien es verdad que la exigencia de notificación previa tiene por objeto abrir una vía de fiscalización general del cumplimiento de todos los requisitos (en particular, de los que vienen previstos por el Anexo III de la Directiva de 2003) que condicionan la validez comunitaria de los Planes, no es menos cierto que uno de los aspectos al que la Comisión Europea presta una atención especial y especifica durante su labor de control es precisamente el del respeto por las autoridades nacionales de las reglas relativas a las ayudas públicas. Por consiguiente, nos parece razonable defender que la obligación de notificación prevista por el TCE en su disciplina de las ayudas públicas estatales queda cumplida con el respeto de la obligación de transmisión a la Comisión Europea de los Planes Nacionales de Asignación que la Directiva impone a los Estados miembros ${ }^{39}$.

por el articulo 88 del TCE, figuran dos posibilidades de actuación particularmente relevantes: en primer lugar, la de acudir al Tribunal de Justicia de la Comunidad Europea en caso de incumplimiento de sus decisiones y, en segundo lugar, la de exigir que los Estados miembros recuperen las ayudas concedidas ilegalmente.

${ }^{38}$ Los Planes se examinan en el seno de un Comité de asistencia a la Comisión Europea creado en virtud del artículo 8 de la Decisión 93/389/CEE del Consejo, de 24 de junio de 1993, relativa a un mecanismo de seguimiento de las emisiones de $\mathrm{CO}_{2}$ y de otros gases de efecto invernadero en la Comunidad. En un plazo de tres meses a partir de la notificación de un Plan por un Estado miembro, como precisa el articulo 9.3 de la Directiva de 2003, la Comisión podrá rechazar dicho Plan, o cualquiera de sus elementos, por razón de su incompatibilidad con el artículo 10 de la misma Directiva de 2003 o con los criterios que figuran en su Anexo III. El Estado miembro sólo tomará una decisión en virtud de los apartados 1 o 2 del artículo 11 si la Comisión acepta las enmiendas propuestas. La Comisión deberá motivar sistemáticamente su decisión de rechazar un Plan o cualquiera de sus elementos.

${ }^{39}$ No es ésta una opinión unánimemente compartida. Algunos autores, en efecto, consideran que se trata de dos trámites distintos, que no pueden equipararse. En tal sentido, por ejemplo, vid. M. Merola y G. Crichlow, «State aid in the framework of the EU position after Kyoto: an analysis of allowances granted under the $\mathrm{CO}_{2}$ emissions allowance trading Directive», World Competition, núm. 27 (1), 2004, pág. 28. Vid. también, E. BERnini y F. RANGHIERI, «Dal Protocollo di Kyoto alla Direttiva europea: il nuovo panorama per l'ordinamento italiano", en vol. col., La nuova direttiva sullo scambio di quote di emissione (a cura di B. Pozzo), Ed. Giuffrè, Milano, 2003, pág. 84. 
b) Un principio general: la incompatibilidad

La clave de bóveda del régimen comunitario de las ayudas estatales reside en la afirmación por el apartado primero del artículo 87 del TCE del principio general de su incompatibilidad con el Mercado común y, por tanto, de la prohibición inicial de su dispensación por los Poderes públicos nacionales. Los fines perseguidos por la Comunidad (artículo 2 del TCE) y los medios que se consideran necesarios para alcanzarlos (artículo 3 del TCE) arrojan luz acerca de cuál sea el fundamento de este pronunciamiento básico de los redactores del Tratado. Mas precisamente, un breve examen de dichos aspectos teleológicos e instrumentales de la construcción comunitaria basta para poner en evidencia que el requisito de la libre competencia es una condición indispensable para la integración económica europea. En consecuencia, ante los efectos "irremediablemente" perturbadores de los mecanismos del mercado achacables a las ayudas (no olvidemos que uno de sus efectos más negativos en el plano económico es el de falsear el funcionamiento normal de la libre y leal competencia), no puede extrañar tal pronunciamiento prohibitivo. Además, es evidente que la finalidad de integración no es solamente económica sino también jurídica y política, lo que explica que el ordenamiento jurídico-comunitario trate de evitar la rivalidad entre los Estados miembros (y, dentro de ellos, entre los distintos Entes territoriales) que podría derivarse de una no deseable "carrera de las subvenciones".

La conclusión lógica que cabe extraer de ello es que, en principio, como se desprende del artículo 9.3 de la Directiva de 2003, los Planes que contengan elementos característicos de las ayudas públicas (en el sentido del artículo 87.1 del TCE y de la premisa antes aludida) deberán ser rechazados por la Comisión Europea por razón de su incompatibilidad con los criterios que figuran en el anexo III de la citada Directiva, al menos en la parte correspondiente, y, también, por incidir en la competencia y en los intercambios entre Estados miembros ${ }^{40}$.

c) Unas excepciones susceptibles de amparar la validez de los Planes

Hemos de tener en cuenta, sin embargo, que la regla general de la incompatibilidad con el Derecho comunitario europeo de las ayudas públicas es-

\footnotetext{
${ }^{40}$ Hemos de señalar, como se desprende del artículo 9.3 de la Directiva de 2003, que la Comisión debe motivar sistemáticamente su decisión de rechazar un Plan o cualquiera de sus elementos.
} 
tatales no es absoluta sino que, como se desprende de los apartados segundo y tercero del antes citado artículo 87 del TCE, existe una serie de excepciones. En efecto, precisamente porque las ayudas estatales pueden revelarse indispensables para conseguir diversos objetivos socio-económicos, dicha regla general no excluye que las que promuevan la realización de fines de interés general perseguidos por el TCE puedan ser declaradas compatibles con el Mercado común. A tal efecto, el resto del artículo 87 del TCE establece un catálogo de supuestos que, en el apartado segundo, responden a las características de excepciones ipso iure (o "incondicionadas") al principio de incompatibilidad y que, en el apartado tercero, pueden considerarse como excepciones "condicionadas", en cuanto, para ser declaradas compatibles, las ayudas contempladas deben cumplir ciertos requisitos.

a') Dos opciones en la elección de la excepción

En principio, cabe considerar que las ayudas que pudieran detectarse en un Plan Nacional de Asignación son susceptibles de tener encaje en dos de las categorías previstas por el antes citado apartado tercero del artículo 87 del TCE, es decir, como se desprende de lo que antes hemos dicho, dos categorías de excepciones "condicionadas" al principio de incompatibilidad" ${ }^{41}$. Nos referimos aquí a las que se establecen en las letras b) y c) de dicho precepto, esto es, respectivamente, a "las ayudas para fomentar la realización de un proyecto importante de interés común europeo" (letra b) y a "las ayudas destinadas a facilitar el desarrollo de determinadas actividades o de determinadas regiones económicas, siempre que no alteren las condiciones de los intercambios en forma contraria al interés común" (letra c).

Un dato importante de la praxis comunitaria que conviene no perder de vista es que la primera clase de excepciones -la que viene prevista por la letra b) - ha dejado de ser el fundamento prioritario de la autorización por parte de la Comisión Europea de proyectos de ayudas estatales "medioambientales" o relacionadas directa o indirectamente con la protección ambiental ${ }^{42}$. Ahora

\footnotetext{
${ }^{41}$ Todo ello sin perjuicio de la posibilidad que ofrece la letra e) del precepto citado. En efecto, nada impide a la Comisión y al Consejo decidir inicialmente que dichas ayudas son compatibles con el mercado común, sin necesidad de reconducirlas a ninguna de las clases de ayudas contempladas expresamente por los apartados segundo y tercero del artículo 87 del TCE. La letra mencionada se refiere literalmente a "las demás categorías de ayudas que determine el Consejo por decisión, tomada por mayoría cualificada, a propuesta de la Comisión”.

${ }^{42}$ Inicialmente, en las decisiones de la Comisión Europea, la letra b) del artículo 87.3 del TCE se interpretaba como el único fundamento legitimador de las ayudas estatales medioambientales.
} 
bien, a pesar de todo, no se puede excluir su aplicación. Prueba de ello es que dicha categoría figura junto con la otra -la de la letra c)- como posible "fundamento de la excepción para todos los proyectos examinados por la Comisión" en la Comunicación que, precisamente, contiene las "Directrices comunitarias sobre ayudas estatales a favor del medio ambiente" (2001/C 37/03) ${ }^{43}$, un texto que establece el régimen aplicable a dichas ayudas hasta el $31 \mathrm{de}$ diciembre de $2007^{44}$. En efecto, si bien es verdad que la Comunicación, en su

Vid. a este respecto, por ejemplo, P. THIEFFRY, La protection de l'environnement, la liberté de commerce et la concurrence, Jurisclasseur Environnement, 1994, Cahiers de Droit des Entreprises, núm. 2, pág. 18. Vid. también, N. DE SADELEER, Le droit communautaire et les déchets, Ed. LGDJ-Bruylant, París-Bruxelles, 1995, pág. 197. En efecto, se consideraba que la contaminación constituía una amenaza común y que, por tanto, la protección ambiental formaba parte de las acciones destinadas a "fomentar la realización de un proyecto importante de interés común europeo". Ténganse presentes los décimo (1980) y vigésimo (1990) Informes sobre la Política de la competencia, en sus puntos 223 y 284 respectivamente.

${ }^{43}$ Esta Comunicación se encuentra publicada en el Diario Oficial de la Comunidad Europea de 3 de febrero de 2001, serie C, núm. 37. Para un comentario de su contenido, vid. por ejemplo, B.F. MACERA y M.Y. FERNÁNDEZ GARCÍA, "El nuevo régimen jurídico-comunitario de las ayudas estatales a favor del medio ambiente", Noticias de la Union Europea, núm. 219, abril 2003, págs. 75 y ss.

${ }^{44}$ Conviene recordar que dicho régimen estaba destinado en sus orígenes (1974) a no mantenerse vigente más de seis años (hasta 1980). Sin embargo, ante los problemas de adaptación de las empresas comunitarias, los textos correspondientes fueron prorrogándose en varias ocasiones. La Comunicación de la Comisión de 6 de noviembre de 1974 "sobre ayudas estatales en favor del medio ambiente", publicada en una carta de 7 de noviembre de 1974 dirigida a los Estados miembros (IVo Informe sobre la Política de la competencia, puntos 175-182), fue prorrogada con algunas modificaciones en 1980 (carta de la Comisión de 7 de julio de 1980; X⿳o Informe sobre la Política de la competencia, puntos 222-226) y en 1986 (carta de la Comisión de 23 de marzo de 1987; XVIo Informe sobre la Política de la competencia, punto 259). Estas directrices, cuya aplicación estaba prevista inicialmente hasta 1992, se prorrogaron un año más (cartas de la Comisión de 18 de enero y de 19 de julio de 1993). Para un resumen de esta evolución, vid. S. Johnson y G. CORCELLE, L'autre Europe verte: la politique communautaire de l'environnement, Ed. Nathan-Labor, París-Bruxelles, 1987, págs. 321-327; M.L. HUIDOBRO, "Instrumentos de política industrial en relación con las actividades medioambientales”, Economia Industrial, núm. 271 (1990), págs. 65-67. Las directrices aprobadas por la Comunicación de 1994 (Diario Oficial de la Comunidad Europea serie C núm. 72, de 10 de marzo de 1994) debían expirar inicialmente el 31 de diciembre de 1999. Sin embargo, el 22 de diciembre de 1999, la Comisión decidió prorrogar su vigencia hasta el 30 de junio del 2000 (Diario Oficial de la Comunidad Europea serie C núm. 14, de 19 de enero del 2000). El 28 de junio del mismo año, la Comisión decidió ampliar la prórroga hasta el 31 de diciembre del 2000 (Diario Oficial de la Comunidad Europea serie C núm. 184, de 1 de julio del 2000). Para un análisis detenido de dichas directrices, vid. B.F. MACERA, El deber industrial..., cit., págs. 356 y ss. Con anterioridad, sobre la evolución del régimen de las ayudas estatales en favor del medio ambiente desde la aprobación por la OCDE de la importante Recomendación 
punto 72, establece que, en principio, "dentro de los limites y en las condiciones fijadas en las presentes Directrices, la Comisión autorizará las ayudas a favor del medio ambiente de conformidad con las disposiciones de la letra c) del apartado del artículo 87 del Tratado CE", el mismo texto afirma también, en el punto siguiente, que " $L a$ excepción de la letra b) del apartado 3 del artículo 87 del Tratado CE podrá aplicarse a las ayudas destinadas a fomentar la realización de proyectos importantes de interés común europeo en ámbitos en que la protección del medio ambiente sea prioritaria y que, por lo general, puedan tener efectos beneficiosos allende las fronteras del Estado miembro $o$ de los Estados miembros interesados" ${ }^{45}$. Es más; consideramos que los objetivos que persigue la implantación por la Directiva de 2003 del sistema de cuotas se acercan más a las preocupaciones que encierra la segunda modalidad de excepciones -letra b) - que a las de la primera -letra c)-. La Directiva de 2003 pretende poner en marcha un auténtico proyecto de interés común europeo que, además, reviste un carácter prioritario evidente y que tiene unas repercusiones que van mucho más allá de las fronteras comunitarias. Y es ésta, al menos según entendemos, la opinión de la Comisión Europea. Dicho órgano autorizó en el año 2002 el primer sistema británico de cuotas de emisión sobre la base de la letra c) del artículo 87.3 del TCE, alegando que dicho sistema no podía beneficiarse de la aplicación de la letra b) del precepto citado por la simple razón de que su modo de funcionamiento y establecimiento se alejaba del que ha de resultar de la transposición de la Directiva y, por tanto, de los rasgos generales de un auténtico proyecto europeo de interés común ${ }^{46}$. De ello podemos deducir, sin riesgo de equivocarnos, que, en el supuesto en que las autoridades comunitarias detecten elementos característicos de ayudas públicas en los Planes nacionales de asignación de cuotas establecidos en el marco de las exigencias de la Directiva de 2003, éstos, a primera vista, podrían autorizarse, en determinadas circunstancias, con arreglo a la excepción contemplada por la letra b) del artículo 87.3 del TCE.

C.74.223 (sobre el principio "quien contamina paga”), vid. E. Alonso García, El Derecho ambiental de la Comunidad Europea, vol. I, Ed. Civitas, Madrid, 1993, págs. 82-85; H. SMETS, "Le principe pollueur-payeur, un principe économique érigé en principe de droit de l'environnement?", Revue Générale de Droit International Public, núm. 2 (1993), pág. 342. Sobre el contenido del texto aquí mencionado, vid. L. IDOT, "Environnement et droit communautaire de la concurrence", Jurisclasseur, núm. 3852, págs. 257 y ss.

${ }^{45} \mathrm{El}$ citado punto 73 sigue rezando que "Es también imprescindible que la ayuda sea necesaria para realizar un proyecto específico y detallado, importante en términos cualitativos y que constituya una contribución ejemplar y verificable al interés común europeo".

46 Nos referimos aquí a la Decisión de 28 de noviembre de 2001 relativa a la ayuda núm. N 416/2001 (Diario Oficial de la Comunidad Europea serie C de 12 de abril de 2002). 
Hemos de puntualizar que el hecho de que nos pronunciemos nítidamente por una de las alternativas planteadas no significa, sin embargo, que debamos prescindir de la otra en nuestro análisis de la posible justificación de Planes nacionales con elementos característicos de ayudas públicas. La excepción contemplada por la letra c) del artículo 87.3 del TCE, como antes hemos dicho, sigue siendo una solución viable en dicha problemática.

b') Otras dos posibilidades en su instrumentación y valoración

Si no existe una única opción clara a la hora de reconducir las ayudas públicas que pudieran existir en los sistemas de asignación a cláusulas justificadoras previstas en el TCE, tampoco la hay en cuanto se refiere al tema de su instrumentación y valoración. En efecto, como se desprende del título del presente epígrafe, caben aquí otras dos posibilidades. La primera, si consideramos que las ayudas en cuestión encajan en la definición de "ayudas estatales a favor del medio ambiente" que nos da la Comunicación de la Comisión (2001/C 37/03) que lleva precisamente este mismo título, a la que ha hemos hecho referencia páginas atrás, es la de aplicar las Directrices específicas que contiene dicho texto ${ }^{47}$. La segunda alternativa es la de valorar directamente tales ayudas públicas sobre la base del artículo 87.3 del TCE, es decir, sobre la base de su letra b) o c), en función de la cláusula justificadora por la que se opte. Esta cuestión dista mucho de ser baladí pues ambas posibilidades presentan diferencias importantes desde el punto de vista de los criterios susceptibles de ser utilizados en la valoración de la compatibilidad o incompatibilidad de las ayudas públicas correspondientes con el Derecho comunitario europeo. Mientras la Comunicación de la Comisión ofrece unos criterios de valoración relativamente precisos, la aplicación directa de las letras b) o c) del artículo 87.3 del TCE es mucho más incierta desde esta perspectiva.

Nuestra opinión, sin embargo, es que no es aplicable, en principio, la Comunicación de la Comisión ${ }^{48}$. En efecto, si bien las Directrices que se formulan en ella, como ya hemos explicado, tienen por objeto genérico los dos tipos de ayudas estatales de los que venimos hablando -previstos por las letras b) y c) del artículo 87.3 del TCE-, no creemos, desde una perspectiva general, salvo excepciones, que las ayudas susceptibles de detectarse en los Planes de asignación de cuotas puedan encajar específicamente en alguna de las dos moda-

\footnotetext{
${ }^{47}$ Diario Oficial de la Comunidad Europea de 3 de febrero de 2001, serie C, núm. 37.

48 Vid. en contra, por ejemplo, S. Rousseaux, "La compatibilité...”, cit., págs. 4 y ss.
} 
lidades ("ayudas de funcionamiento" y "ayudas a la inversión”) previstas en dicho texto. Por un lado, la Comunicación no contempla más que cuatro categorías de ayudas de funcionamiento: las ayudas a favor de la gestión de residuos y del ahorro energético ${ }^{49}$, las ayudas en formas de desgravaciones y exenciones fiscales ${ }^{50}$, las ayudas a favor de las energías renovables ${ }^{51} \mathrm{y}$, finalmente, las

\footnotetext{
${ }^{49}$ Las ayudas de funcionamiento en favor de la gestión de residuos y del ahorro energético han de limitarse a la estricta compensación de los costes de producción adicionales en relación con los precios de mercado de los productos o servicios correspondientes. Además, deben ser temporales y, en principio, decrecientes (punto 43). En el caso de las ayudas decrecientes, la duración máxima será de cinco años y su intensidad podrá ser del $100 \%$ de los costes adicionales el primer año, pero deberá reducirse de manera lineal hasta alcanzar el tipo cero al final del quinto año (punto 45). En el caso de las ayudas no decrecientes, su duración se limitará también a cinco años y su intensidad al $50 \%$ de los costes adicionales (punto 46).
}

${ }^{50}$ Las ayudas de funcionamiento en forma de desgravaciones y exenciones fiscales, como su nombre indica, se destinan a introducir excepciones temporales que disminuyen la carga fiscal de determinadas empresas en relación con los impuestos establecidos para gravar ciertas actividades por motivos de protección medioambiental, en particular cuando no hay armonización a nivel europeo o cuando existen riesgos de pérdida de competitividad internacional de dichas empresas (punto 47). En el supuesto en que el establecimiento del impuesto obedezca a una Directiva comunitaria, las ayudas de este tipo pueden autorizarse cuando el Estado miembro aplica un tipo superior al mínimo prescrito por la Directiva para determinados productos y concede excepciones a determinadas empresas que pagarán así un tipo inferior, pero al menos igual al mínimo establecido por la Directiva. Si el Estado aplica un impuesto al tipo mínimo prescrito por la Directiva, las excepciones para determinadas empresas podrán autorizarse únicamente en el caso en que la Directiva en cuestión contemple la posibilidad de su existencia. Cuando un Estado miembro introduzca un impuesto nuevo o cuando el impuesto contemplado por el Estado miembro sea superior al impuesto establecido por la norma comunitaria, la exención será de diez años de duración, sin posibilidad de reducción gradual. Conviene tener en cuenta que dichas disposiciones pueden aplicarse a los impuestos existentes si el impuesto en cuestión produce une efecto ambiental positivo y, sobre todo, si las excepciones en favor de las empresas beneficiarias se han establecido en el momento de adopción del impuesto o son necesarias como consecuencia de una modificación significativa de las condiciones económicas que coloque a las empresas en una situación competitiva difícil (punto 51). En este último supuesto, el importe de la reducción fiscal no puede ser superior al incremento de cargas derivado de la modificación de las condiciones económicas. Cuando desaparezca este aumento de cargas, también deberá desaparecer la reducción fiscal. Los Estados miembros pueden asimismo fomentar la puesta a punto de procesos de generación de electricidad a partir de la energía tradicional que permitan alcanzar una eficacia energética sensiblemente superior a la obtenida con los procesos tradicionales. En tal supuesto, la Comisión estima que las exenciones totales de impuestos pueden estar justificadas por un periodo de cinco años, en ausencia de reducción gradual. También se pueden conceder excepciones de diez años, con arreglo a las condiciones antes mencionadas.

${ }^{51}$ Las ayudas en favor de las energías renovables pueden resultar necesarias cuando los procedimientos técnicos disponibles no permitan producir la energía en cuestión a un coste unitario comparable con el de las energías tradicionales (punto 55). En realidad, dichas ayudas pueden justificar- 
ayudas para la producción combinada de electricidad y calor ${ }^{52}$. La asignación de cuotas, si bien puede incitar a las empresas a perseguir objetivos equiparables a los que pretenden alcanzar las ayudas mencionadas (por ejemplo, el

se para sufragar la diferencia entre el coste de producción de la energía generada a partir de fuentes renovables y su precio de mercado (punto 56). La Comunicación contempla esencialmente tres opciones. La primera se refiere a las ayudas estatales destinadas a compensar la diferencia entre los costes de producción de las energías renovables y el precio de mercado de la electricidad (punto 59). Estas ayudas de funcionamiento sólo podrán concederse, en su caso, para garantizar la amortización de las instalaciones. Los kwh adicionales que produzca la instalación en cuestión no podrán beneficiarse de ninguna ayuda. No obstante, la ayuda también podrá cubrir los gastos financieros y una remuneración justa del capital (punto 59). La segunda opción es la de las ayudas estatales que se conceden bajo la forma de lo que las Directrices llaman "mecanismos de mercado", como, por ejemplo, los certificados verdes o los sistemas de licitación (punto 61). En el supuesto en que estos sistemas constituyan ayudas estatales, pueden ser autorizados por la Comisión Europea siempre que los Estados miembros puedan demostrar que la ayuda es indispensable para garantizar la viabilidad de las energías renovables, que, en conjunto, no entraña una compensación excesiva en beneficio de las energías renovables y que no disuade a los productores de estas energías de incrementar su competitividad. Para verificar si se cumplen dichos criterios, la Comisión Europea tiene la intención de autorizar estos sistemas de ayuda durante periodos de diez años. Al término del periodo de diez años, se deberá hacer balance para determinar si es necesario proseguir con la ayuda (punto 62). La tercera opción, por último, hace referencia a las ayudas de funcionamiento de las que se benefician las nuevas instalaciones de producción de energía renovable, calculadas sobre la base de los costes externos evitados (punto 70). Los costes externos evitados son los costes medioambientales que la sociedad debería soportar si la misma cantidad de energía se generase en una instalación de producción que funcionase con energías convencionales. Estos costes se calculan sobre la base de la diferencia entre, por un lado, los costes externos producidos y no abonados por los productores de energía renovable y, por otro, los costes externos producidos y no abonados por los productores de energía no renovable. El importe de la ayuda concedida al generador de energía renovable no podrán exceder de 5 céntimos de euro por kwh. Conviene señalar en relación con esta opción que la Comisión Europea ha de estar segura de que la ayuda no provoca un falseamiento de la competencia contrario al interés común. En otras palabras, dicho órgano debe tener la certeza de que la ayuda logrará un desarrollo global efectivo de las energías renovables en detrimento de las convencionales y no una simple transferencia de cuotas de mercado entre energías renovables. Más precisamente, se deben cumplir las condiciones siguientes: 1) la ayuda ha de formar parte de un sistema que ofrezca igualdad de trato a las empresas del sector de las energías renovables; 2) el sistema ha de contemplar la concesión de ayudas sin discriminación entre las empresas que producen la misma energía renovable; 3) este sistema ha de ser controlado por la Comisión Europea cada cinco años (punto 64).

${ }^{52}$ Las ayudas de funcionamiento para la producción combinada de electricidad y calor pueden justificarse si se demuestra que son útiles para la protección medioambiental y cuando los costes de producción de electricidad o calor sean superiores a los precios del mercado (punto 66). Como precisa el punto 67 de las Directrices, se pueden conceder en las mismas condiciones ayudas en favor de la utilización industrial de la producción combinada de calor y electricidad, si se demuestra que el coste de producción de una unidad de energía mediante esta técnica es superior al precio de mercado de una unidad de energía tradicional. 
ahorro energético), no puede integrarse directamente en ninguna de estas categorías. Por otro lado, las ayudas a la inversión que son susceptibles de acogerse a las Directrices son exclusivamente las que permiten a las empresas superar las normas comunitarias vigentes ${ }^{53} \mathrm{y}$, también, las que vayan dirigidas a centros productivos que realicen inversiones en ausencia de normas comunitarias obligatorias o que deban hacerlo para adaptarse a normas nacionales más estrictas que las normas comunitarias vigentes ${ }^{54}$. La asignación de cuotas, en principio, salvo que se den circunstancias específicas en determinados Estados miembros, no tiene ninguno de estos propósitos. No es más que un instrumento destinado a facilitar el cumplimiento de la legislación comunitaria sobre emisiones de gases de efecto invernadero, que refleja, como ya hemos explicado páginas atrás, lo que venía o vendría definido por normas o por actos administrativos de policía en el marco de un esquema clásico de protección ambiental basado en la coacción. Además, como hemos afirmado al contrastar los elementos característicos de las ayudas públicas con el sistema de asignación y como reconoce la propia Comisión Europea -reiteramos aquí su pronunciamiento-, "el comercio de derechos de emisión no implica nuevos objetivos ambientales sino que facilita el cumplimiento menos costoso de los objetivos vigentes" $" 55$.

53 Sólo pueden autorizarse las ayudas hasta un nivel máximo del $30 \%$ bruto del coste de inversión subvencionable (punto 29). Como precisa el punto 37 de la Comunicación, los costes subvencionables se limitan estrictamente a los costes de las inversiones adicionales realizadas para alcanzar los objetivos de protección ambiental. En todos los casos, los costes subvencionables han de calcularse netos de las ventajas obtenidas de un eventual incremento de la capacidad, de los ahorros de costes generados durante los cinco primeros años de vida de la inversión y de las producciones accesorias adicionales durante el mismo periodo.

${ }^{54}$ En caso de adaptación a normas nacionales adoptadas en ausencia de normas comunitarias, los costes subvencionables incluyen el coste de las inversiones adicionales para alcanzar el nivel de protección ambiental exigido por las normas nacionales. En caso de adaptación a normas nacionales más estrictas que las normas comunitarias o en caso de superarse voluntariamente las normas comunitarias, los costes subvencionables incluyen el coste de las inversiones adicionales para alcanzar un nivel de protección ambiental superior al exigido por las normas comunitarias. No son subvencionables los costes vinculados a las inversiones realizadas para alcanzar el nivel de protección exigido por las normas comunitarias. Finalmente, en ausencia de normas, los costes subvencionables incluyen el coste de las inversiones necesarias para alcanzar un nivel de protección ambiental superior al que alcanzarían las empresas de que se trate en ausencia de toda ayuda estatal en favor del medio ambiente (punto 37).

55 Preguntas y respuestas..., cit., punto 1 . 
Es posible que la próxima modificación de estas Directrices ${ }^{56}$ conlleve la inclusión, en su ámbito de aplicación, de las cuotas de emisión, como posible forma de ayudas públicas. En el caso en que así sea, aunque no existe ninguna garantía de ello ${ }^{57}$, es evidente que nuestro planteamiento dejará de ser válido y dichas Directrices deberán aplicarse al supuesto que estamos analizando. Mientras tanto, sin embargo, en nuestra opinión, como ya hemos explicado, debemos seguir considerando que las ayudas susceptibles de detectarse en los Planes nacionales de asignación, en principio, han de valorarse directamente sobre la base del artículo 87.3 del TCE; en su letra b), si las contemplamos como ayudas para la realización de un proyecto importante de interés común europeo (alternativa por la que optamos), o en su letra c), si interpretamos que se trata de ayudas destinadas a facilitar el desarrollo de determinadas actividades.

Tal y como antes hemos señalado, no existen criterios estrictos de interpretación de las letras antes citadas, sino que la Comisión tiene un amplio margen de discrecionalidad para declarar compatibles con el Mercado común las categorías de ayudas previstas en ellas ${ }^{58}$. De nuevo, tenemos que acudir a la jurisprudencia del TJCE para encontrarnos con algunas pautas. Más allá del requisito de la proporcionalidad (o justificación compensatoria), una de las más importantes es, sin duda, la que se formula a partir de la diferencia de tratamiento que reserva el TCE a cada una de las dos categorías de excepciones aquí contempladas. Más precisamente, se establece que las ayudas comprendidas en la letra b) del artículo 87.3 "podrán considerarse compatibles con el mercado común", sin más, mientras que las ayudas contempladas en la letra c) sólo "podrán considerarse compatibles con el mercado común" si no alteran "las condiciones de los intercambios en forma contraria al interés común". De ello se puede deducir que, si bien no se puede obviar la necesidad de hacer un balance en todos los

\footnotetext{
${ }^{56}$ Recordaremos que el plazo de vigencia de las Directrices actuales acabará el 31 de diciembre de 2007.

${ }^{57}$ Conviene advertir que esta ampliación del ámbito de aplicación de las Directrices ya se venía adelantando antes de su última modificación (en los años 2000-2001). Vid. por ejemplo, J. LEFEVERE, "EC trade and competition law sigues raised by the design o fan EC emissions trading system", Foundation for International Environmental Law and Development, London, junio 1999, pág. 18. Vid. también, S. Rousseaux, "La compatibilité...", cit., pág. 10.

${ }^{58}$ El Tribunal de Justicia de la Comunidad Europea viene reiterando esta advertencia con mucha frecuencia, desde la famosa Sentencia de 17 de septiembre de 1980, "Philip Morris Holland BV c/. Comisión".
} 
supuestos entre los efectos beneficiosos de las ayudas que puedan detectarse en los Planes nacionales de asignación y sus efectos distorsionadores de los competencia ${ }^{59}$, resultará menos costoso justificar su compatibilidad con el mercado común si consideramos que se trata de "ayudas para fomentar la realización de un proyecto de interés común europeo".

${ }^{59}$ Cabe considerar, en efecto, como se desprende de la Sentencia del Tribunal de Justicia de la Comunidad Europea recaída en el asunto "Francia c/. Comisión" (núm. 47/69), que las distorsiones de la competencia que excedan de un determinado límite son contrarias al interés común, con independencia de que los efectos que puedan tener las medidas que originan las distorsiones con respecto a otros objetivos comunitarios. 